\title{
The effect of molding conditions on the quality of geopolymer surfaces
}

\author{
JANUSZ JAGLARZ ${ }^{1}$, DoMinik WYSZYŃSKI ${ }^{2 *}$, Michą LACH ${ }^{1}$, JANUSZ MIKUŁA $^{1}$, RYSZARD DURAJ ${ }^{3}$ \\ ${ }^{1}$ Institute of Material Engineering, Cracow University of Technology, \\ al. Jana Pawła II 37, 31-864 Cracow, Poland \\ ${ }^{2}$ Institute of Production Engineering, Cracow University of Technology, \\ al. Jana Pawła II 37, 31-864 Cracow, Poland \\ ${ }^{3}$ Institute of Physics, Cracow University of Technology, \\ ul. Podchorążych 1, 30-084 Cracow, Poland
}

${ }^{*}$ Corresponding author: pujaglar@cyfronet.pl

The presented work describes the method of measuring surface topography with application of BRDF (bidirectional reflectance distribution function), ellipsometry and spectrophotometry. This non-contact method enables measurement and analysis of large area surfaces, such as plasters and facades. A standard method of topography analysis does not describe sufficiently all of the interesting features. The visual aspect of the surface evaluation is very important from the functional and utilitarian point of view. The proposed methods of surface analysis enable not only the quantitative evaluation but also indirectly the qualitative properties (visual aspects).

Keywords: surface topography, bidirectional reflectance distribution function, ellipsometry, spectrophotometry.

\section{Introduction}

Geopolymers are a novel class of materials that are formed in strongly alkaline environments in process called geopolymerization [ $[\underline{-} \underline{3}]$. The final product of this process is an amorphous three-dimensional framework structure [ $\underline{1}-\underline{5}]$. Geopolymers are based on aluminosilicates, both natural and synthetic (e.g. metakaolin, fly ash, blast furnace slag, etc.) $[\underline{1}, \underline{2}, \underline{4}]$. Geopolymers are in the field of interest of many industries. Their numerous applications are confirmed, including: insulating panels and wall elements, decorative stones, low-tech building materials, foamed materials (for thermal insulation), imitating ceramic slabs, fire retardant materials, equipment for the foundries, aerospace (civil and military) and parts for cars, matrix composites, adhesives and resins (including high-tech technology), matrices to immobilize hazardous and radioactive wastes, but above all, unlimited use as cements replacement in civil engineering [ $\underline{1}-\underline{8}]$. The number of applications continues to grow - huge amount of them has been confirmed by dozens 
of patents and hundreds of publications all around the world. Depending on the needs, it is possible while producing geopolymers, to achieve a perfect surface smoothness, for instance by using suitable molds. Those geopolymers not only meet the strict mechanical properties, but they also meet the aesthetics criterion, appearance and visual look, which make them better than other human-made materials and comparable only with natural stone. The results in this study show that the surface smoothness of molds has the greatest impact of the quality of the surface of geopolymers achieved. There are many geopolymer applications in various industries. This study shows a variety of applications of geopolymers, starting from the typical building materials through chimney pipes and ending with furniture and even garden architecture. In the study, the authors used a variety of optical methods (both standard and non-standard) to examine the surface of selected geopolymers. These methods include: spectroscopic techniques, bidirectional reflection distribution function (BRDF), total integrated scattering (TIS) or even spectroscopic ellipsometry. Optical methods are non-destructive and non-contact, and may be used to determine surface roughness and other topographic parameters in the large area of samples. Also due to the development of numerical methods allowing to simulate reflection from surfaces with very complex topographies, the interest in application of non-invasive and non-contact optical methods for surface studies increased [9]. Parameters such as thickness, roughness, autocorrelation length are strongly influential on surface topography. Those parameters determine how much radiation will be dispersed and how much will be scattered. The shape of these irregularities will also affect the angular distribution of scattered radiation. The main goals of this work are optical descriptions of geopolymer surfaces and determination of macroscopic parameters characterizing films' topography.

\section{Optical methods}

For many years the optical methods (OM) were broadly used for studies of the quality of surfaces. The optical techniques are contactless and noninvasive - so useful for control and monitoring of surface properties. They can be divided into 3 groups.

Group 1. Integrating techniques based on determination of total intensity of scattered radiation (TIS methods) [10]. In the TIS techniques used in surface measurements, the integrating spheres are used. They allow to measure the total $\left(R_{\text {tot }}\right)$ and diffusive $\left(R_{\text {diff }}\right)$ reflectance on studied surfaces. The directional (specular) reflectance is the difference between values of total and diffusive reflectance. By knowing the values of the diffusion and specular components, it is possible to calculate the total integrated scatter (TIS) parameter, which is: TIS $=R_{\text {diff }} / R_{\text {tot }}[11-13]$. The gloss coefficient is simply GL $=1-$ TIS. Using the radiation diffusively reflected from the sample, and comparing it with the radiation specularly reflected from the flat surface, one can determine the surface roughness and other statistical parameters characterizing the quality of surface [12]. However, limitations of TIS method result from the character of angular distribution of scattered light and allow to study only relatively flat samples. Also TIS methods are used for color measurements. The sensitivity of TIS strongly depends on the diam- 
eter of used sphere (Ulbricht ball) and the type of material from which this sphere was made $[\underline{10}, \underline{12}]$.

Group 2. Optical methods are based on the measurements of angular light scattering on studied surfaces. Especially, the BRDF technique is useful to analyze the topography of the samples' surfaces $[\underline{14}, \underline{15}]$. The BRDF may be used to study any material and allows to obtain information about any surfaces. The BRDF determines the angular distribution of the intensity of radiation reflected from the sample. In this method, a single beam of laser radiation falls on the examined surface at the defined angle of incidence $\theta_{\mathrm{i}}$. The scattered light intensity is recorded by a detector for varying angles $\theta_{\mathrm{s}}$. The angular distribution of the intensity of the scattered light contains information about surface topographic parameters such as surface roughness $\sigma$ RMS (root mean square), statistical or periodic distribution of heights. The BRDF function is defined as the power of scattered light $P_{\mathrm{s}}$ per the solid angle of receiver aperture $\Omega$ (acceptance angle $\Omega$ ) in the $\theta_{\mathrm{s}}$ direction and per incident power $P_{\mathrm{i}}$ coming from the $\theta_{\mathrm{i}}$ direction $[\underline{16}, \underline{17}]$, namely:

$$
\mathrm{BRDF}=\frac{P_{\mathrm{s}}}{P_{\mathrm{i}} \Omega \cos \theta_{\mathrm{s}}} \quad\left[\mathrm{sr}^{-1}\right]
$$

The BRDF function is usually represented in the spatial frequency domain $f$. The relation between $f$ and incident angle $\theta_{\mathrm{i}}$, scattered angle $\theta_{\mathrm{s}}$, and light wavelength $\lambda$ is as follows:

$$
f=\frac{\sin \theta_{\mathrm{s}}-\sin \theta_{\mathrm{i}}}{\lambda}
$$

The advantage of optical techniques based on scanning surface is large (average measuring area is usually more than $10^{6} \mu \mathrm{m}^{2}$ ). Such a value is significantly higher than AFM (atomic force microscope) scanning area (average $10^{2} \mu \mathrm{m}^{2}$ ). Therefore topographical parameters determined from optical measurements describe studied surface in the longer spatial wavelength. The BRDF technique allowed to investigate the geopolymer surfaces in lower spatial frequency.

Group 3. Ellipsometric techniques use the phenomenon of changing polarization state of the linearly polarized light after reflection. The change in the polarization state depends on the optical properties and roughness of the sample, the angle of incidence and the wavelength of incident light. In ellipsometry, two angles, $\Psi$ and $\Delta$, are defined in a way that describes the polarization state of the reflected light $[\underline{18}, \underline{19}]$. The tangent of angle $\Psi$ is defined as a ratio of complex Fresnel reflection coefficients $r_{\mathrm{p}}$ and $r_{\mathrm{s}}$ : $\tan \Psi=\left|r_{\mathrm{p}}\right|^{2} /\left|r_{\mathrm{s}}\right|^{2}$, where subscripts 'p' and 's' relate to reflected light perpendicularly and vertically polarized to incident plane, respectively. The $\Delta$ is a phase shift between both polarized waves. Furthermore, reflection coefficients meet the equation $r_{\mathrm{p}} / r_{\mathrm{s}}=$ $=\exp (\mathrm{i} \triangle 4) \tan \Psi$. Through this fundamental equation of ellipsometry one can determine refraction indices $n$ and extinction coefficients $k$ of studied samples. Moreover thanks to knowledge of dispersion of $n$ and $k$ in the wide spectral range, one can determine 
many interesting information about band structures in materials, and their optical and electrical properties. Ellipsometry gives reliable results if the sample surface is smooth enough that it could be possible to determine the optical parameters of the surface (spectral dependences of refractive index $n(\lambda)$ and extinction coefficient $k(\lambda)$ ) by the use of ellipsometry.

\section{Experimental details}

\subsection{Geopolimeric samples}

The geopolymeric mass was obtained by mixing fly ash or metakaolin with sand in a ratio of 1:1 by weight; aqueous activating solution consisting of an aqueous solution of sodium hydroxide, water and aqueous sodium glass (R-145). Metakaolin and flying ash particle size was less than $40 \mu \mathrm{m}$. The entire mass was mixed homogeneously and then put into plastic molds. Forms containing the mass were sealed with plastic foil and put into a laboratory drier for 24 hours at $75^{\circ} \mathrm{C}$. In the next step, the samples were removed from forms and stored for 28 days. Geopolimeric samples, both metakaolinand fly-ash-based were put to two different mold types: polycarbonatic and polypropylenic. Moreover some of them were colored to achieve different surface color for different samples. Chemical composition of used raw materials (fly ash and metakaolin) is shown in Table 1.

\subsection{Optical testing}

The spectroscopic measurements of total reflectance were performed in spectral range 250 to $2500 \mathrm{~nm}$ by the use double beam Perkin Elmer Lamda 900 spectrophotometer equipped with an integrating sphere. To determine color parameters and gloss coefficient, we used the ISP-REF (Avantes company) integration sphere. For recording the total and diffusive reflected light beam from the sample surface, the Avantes spectrometer was used. Although the full spectral range was from 300 to $1100 \mathrm{~nm}$, we present spectra limited to visible part of light (i.e. $360-780 \mathrm{~nm}$ ). The BRDF measurements were performed be means of an automatic scatterometer. It consists of a $650 \mathrm{~nm}$ laser diode as a light source with the beam diameter of $2 \mathrm{~mm}$ mounted on a goniometric table with $0.1 \mathrm{deg}$ resolution. The light scattered at the sample surface was measured with a silicon photodiode detector. The rotations are obtained by a computer controlled step-

$\mathrm{T}$ a b 1 e 1. Chemical composition (\% by mass) of fly ash used (from CHP Coal Power Plant in Skawina) and metakaolin 1 and 2 .

\begin{tabular}{llllllll}
\hline Material & $\mathrm{SiO}_{2}$ & $\mathrm{Al}_{2} \mathrm{O}_{3}$ & $\mathrm{CaO}$ & $\mathrm{Fe}_{2} \mathrm{O}_{3}$ & $\mathrm{MgO}$ & $\mathrm{TiO}_{2}$ & $\mathrm{~K}_{2} \mathrm{O}+\mathrm{Na}_{2} \mathrm{O}$ \\
\hline Fly ash & 56.0 & 23.5 & 2.70 & 5.90 & 2.60 & 1.10 & 4.10 \\
Metakaolin 1 & $51.0-53.0$ & $40.0-42.0$ & $0.25-0.30$ & $1.2-1.3$ & $0.30-0.35$ & $0.7-0.75$ & $1.40-1.45$ \\
Metakaolin 2 & $51.0-53.0$ & $40.0-42.0$ & $0.25-0.30$ & $1.3-1.4$ & $0.35-0.40$ & $0.7-0.75$ & $1.35-1.40$ \\
\hline
\end{tabular}


per motor. For a fixed angle of incidence, the scattered intensity in the plane of incidence has been measured by varying the detector orientation. All measurements have been carried out with the s-polarized incident beam. In any case, the sample surface size was much larger than the beam diameter. The BRDF measurements and calculations have determined the roughness and autocorrelation length of surfaces.

Figures 1 and 2 present SEM pictures where there is no possibility to determine optical properties of the surface - topographic information has a rather qualitative dimension. For the presented samples, the ellipsometric measurements have been performed by a spectroscopic ellipsometer M-2000 manufactured by J.A.Woollam Co. (USA) in the spectral range 190-1700 $\mathrm{nm}$. The samples were measured for four angles of incidence $\left(60^{\circ}, 65^{\circ}, 70^{\circ}, 75^{\circ}\right)$. In one experiment additionally were measured intensities of reflected light and depolarization coefficient, simultaneously [20]. To analyze the data, we combined all angular spectra and we have fitted all the data simultaneously. For better accuracy, the data have been analyzed using CompleteEASE software.

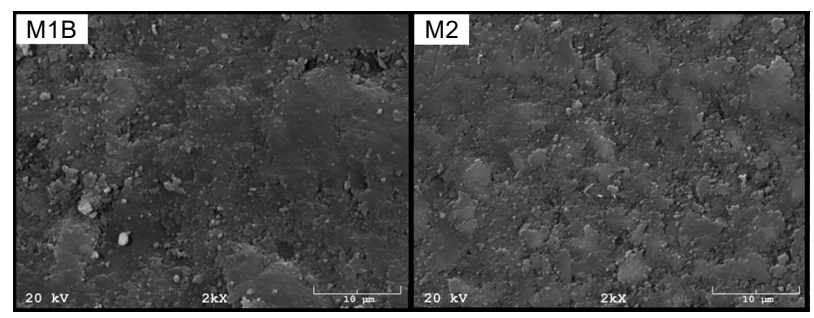

a

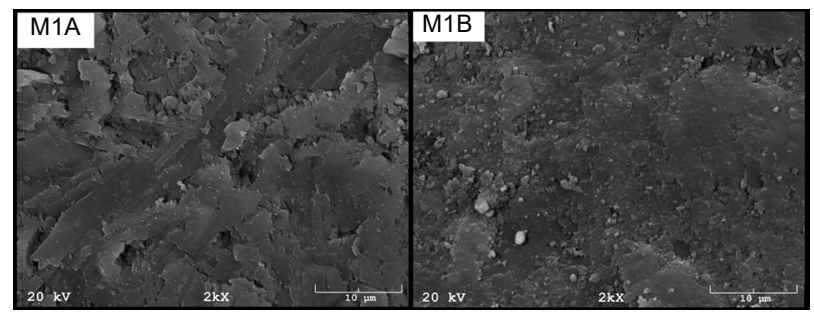

b

Fig. 1. SEM photography of two different metakaolin, the same mold (polypropylene) (a), and the same metakaolin, two different molds (polypropylene and polycarbonate) (b).

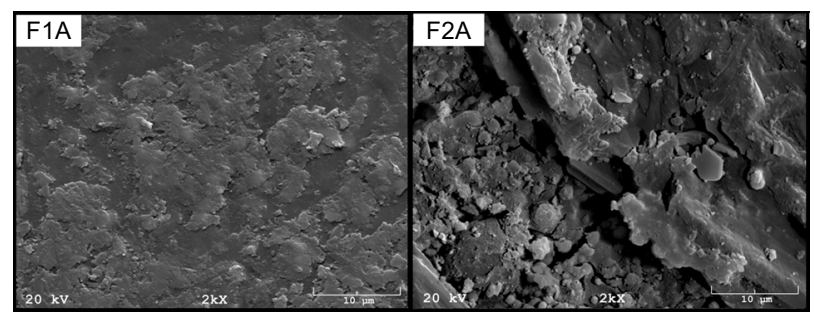

Fig. 2. SEM photography of the same fly ash, two different molds, polypropylene and polycarbonate. 


\section{Results and discussion}

The results presented below concern three types of geopolymers. The investigated surfaces of the geopolymers differed due to the material composition and the mold used to produce them. For better clarity we named samples according to Table 2 .

The samples differed visually. Some samples were tinted with selected dyes, as shown in Table 2. Figure 3 a shows the total reflection of geopolymer samples based on fly ash, and Fig. $3 \mathbf{b}$ based on metakaolin. The presence of dyes caused differences in spectra in the visible light spectrum.

In the VIS range, spectral reflectances differ due to the presence of dyes. For all presented geopolymers, we made color measurements. The values of color parameters determined in $L^{*} a^{*} b$ system are presented in Table 3 .

$\mathrm{T}$ a b 1 e 2. Sample notation, materials used for geopolymer samples, mold material and dye used.

\begin{tabular}{llllllll}
\hline & F1A & F1B & F2A & F2B & M1A & M1B & M2 \\
\hline Base material $^{\mathrm{a}}$ & Fly ash & Fly ash & Fly ash & Fly ash & Metakaolin 1 & Metakaolin 1 & Metakaolin 2 \\
Mold material $^{\text {b }}$ & PC & PC & PP & PP & PC & PP & PP \\
Dye $^{c}$ & - & $\mathrm{Fe}_{2} \mathrm{O}_{3}$ & - & $\mathrm{Cr}_{2} \mathrm{O}_{3}$ & - & - & - \\
\hline
\end{tabular}

${ }^{\mathrm{a}}$ In accordance to Table 1 .

${ }^{\mathrm{b}}$ In experiment two types of molds were used: polycarbonatic (PC) and polypropylenic (PP).

${ }^{\mathrm{c}}$ Dyes were added as powder to fly ashes and dry mixed before adding the alkaline activator.
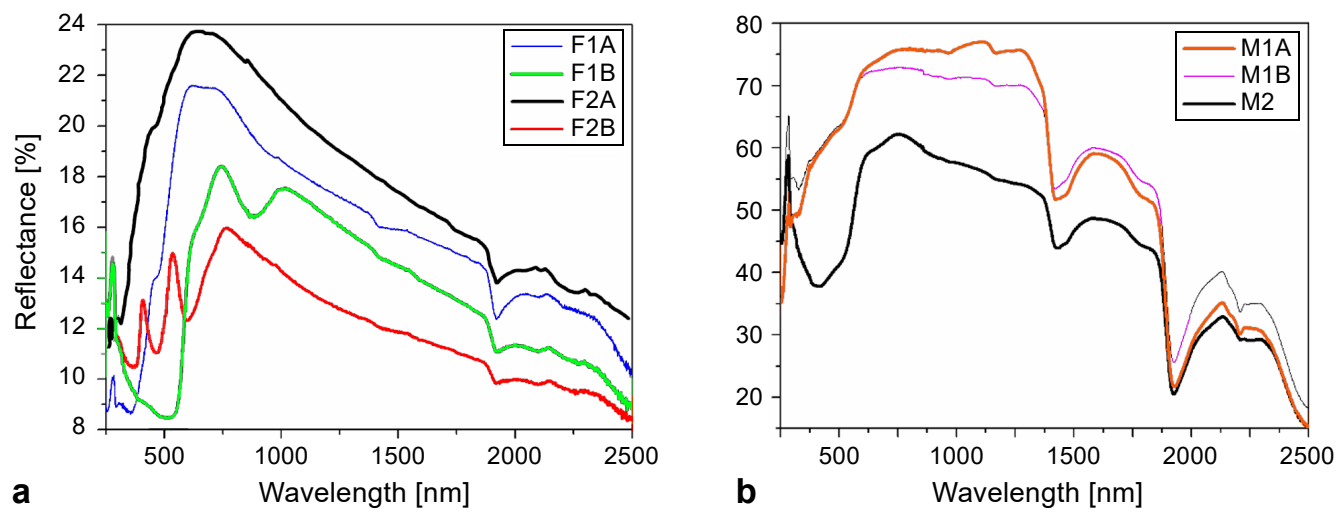

Fig. 3. Spectral total reflectance of geopolymer samples based on flying ashes (a), and metakaolin (b).

$\mathrm{T}$ a b 1 e 3. CIE $\left(L^{*} a^{*} b\right)$ color space parameters. International Commission on Illumination (Commission Internationale de l'Éclairage) elaborated distributions of wavelengths in the electromagnetic visible spectrum and physiologically perceived colors in human color vision.

\begin{tabular}{|c|c|c|c|c|c|c|c|}
\hline & F1A & F1B & F2A & F2B & M1A & M1B & M2 \\
\hline$\overline{a^{*}}$ & 1.8 & 11.68 & 1.49 & -3.84 & 3.79 & 2.59 & 8.74 \\
\hline$b$ & 9.86 & 5.87 & 5.01 & 5.82 & 7.02 & 7.66 & 14.53 \\
\hline$L^{*}$ & 54.44 & 30.42 & 60.9 & 35.48 & 88.34 & 79.25 & 66.49 \\
\hline
\end{tabular}


$\mathrm{T}$ a b 1 e 4. Mean roughness (roughness average $R_{\mathrm{a}}$ ) of surfaces of the samples.

\begin{tabular}{llllllll}
\hline & F1A & F1B & F2A & F2B & M1A & M1B & M2 \\
\hline$R_{\mathrm{a}}[\mu \mathrm{m}]$ & 0.45 & 1.24 & 1.34 & 1.6 & 0.87 & 0.74 & 0.6 \\
\hline
\end{tabular}

T a b 1 e 5 . RMS roughness to $R_{\mathrm{a}}$ conversion table.

\begin{tabular}{|c|c|c|c|c|c|c|c|c|c|c|c|}
\hline$\overline{\mathrm{RMS}}$ & 2200 & 1100 & 550 & 275 & 137.5 & 69.3 & 35.2 & 17.6 & 8.8 & 4.4 & 2.2 \\
\hline$\overline{R_{\mathrm{a}}[\mu \mathrm{m}]}$ & 50 & 25 & 12.5 & 6.3 & 3.2 & 1.6 & 0.8 & 0.4 & 0.2 & 0.1 & 0.05 \\
\hline
\end{tabular}

Naturally dye admixtures do not affect the spectral response of reflectance for wavelength in the range of infrared light. As may be seen in Fig. 3 for wavelengths $\lambda$ longer than $800 \mathrm{~nm}$, the total reflectance basically depends on main geopolymers' raw materials, i.e. fly ashes and metakaolin. As may be concluded, the UV-VIS-NIR analysis could be used to pre-evaluate the chemical composition of geopolymers. Their optical spectra are very similar to ones in literature. As may be noticed in Fig. 3, the reflectance minima observed in metakaolin and flying ashes reflectance spectra have the same values for the wavelength $1920 \mathrm{~nm}$. Probably this is due to similarity between the chemical and structural structure of both materials. The values of surface roughness (RMS) obtained from mechanical profilometry measurements have been presented in Table 4. The conversion table was presented in Table 5. As may be concluded, the topographies of geopolymer (GP) surfaces differ. As may be noticed, the roughness varies and depends on the used mold. However, they are less dependent on GP material. It confirms our prediction. Namely GP materials were obtained in similar manufacturing processes, the scattering of reflected light from the surface of the GP does not substantially depend on the chemical composition and structure of the atomic bonds occurring in GP bulk. The diffusive (non-directional) scattering results from the surface roughness which depends on the type of used mold.

As presented above, the lowest roughnesses have the samples F1A, which were fly -ash-based geopolymer samples obtained in polycarbonatic molds without any dyes. For fly ash-based samples lower roughness values were achieved for samples obtained in polycarbonatic molds. However the opposite effect was observed for metakaolin -based samples, where lower roughness values were lined to polypropylenic molds. Generally, metakaolin-based samples have lower roughness with only one exception, sample F1A. Additionally, geopolymer surfaces present a small gloss effect. Gloss coefficient is often used to characterize the gloss ratio. It is equal to the ratio of light intensity reflected to the total light scattered from the surface of the sample [10]. Figure 4 shows spectra of gloss coefficients of geopolymer samples based on flying ashes (Fig. 4a) and metakaolin (Fig. 4b).

The major advantage of the determining gloss coefficient is that the decisive factor affecting its value is only the surface topography, not the phase and chemical composition. For metakaolin-based samples, it could be observed that curves shown in Fig. $4 \mathbf{b}$ are almost identical, regardless of the used mold. Probably the value of the 

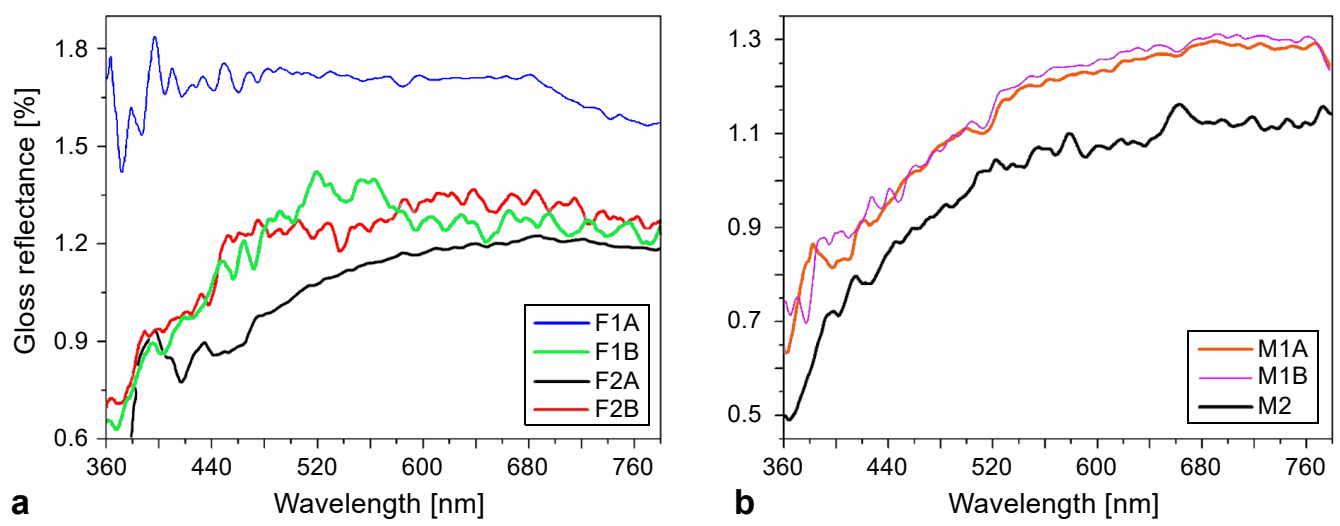

Fig. 4. Gloss parameter for geopolymer samples based on flying ashes (a) and metakaolin (b).

gloss ratio is not influenced by the applied form or its effect is negligible. The spectra for samples based on two different metakaolins were similar in shape, but for the second metakaolin (M2) the gloss values were smaller. This proves that the structure of the material is significantly affected by the gloss coefficient. An additional confirmation of this are graphs in Fig. 4a. Gloss coefficients for fly-ash-based geopolymers vary in both shape and value with those based on metakaolin. As may be concluded, the addition of dyes to fly-ash-based geopolymers caused a reduction in the gloss coefficient. Generally, according to our intuition, GP surfaces are more glossy when surface is flatter. In the next step, we chose sample F1A for ellipsometric measurement due to the largest value of the gloss coefficient. As a matter of fact, the specular reflection occurs when the partly coherent light is appearing in reflected light. The Fresnel model based on the coherence theory allows to determine optical parameters ( $n$ and $k$ ) from ellipsometric data if the depolarization degree is less than $80 \%$. This condition was fulfilled for sample 1 throughout the spectral range of the M2000 ellipsometer. The ellipsometric studies were done for sample F1A. The directionally reflected beams measured in ellipsometry consist of two parts. One was polarized light with two reflecting parameters. First reflectance obeys the Fresnel law of directional reflection and the second one were noncoherent and depolarised reflected beams. The ratio of intensity of coherently reflected light to incoherent light component is defined as depolarization degree or coefficient. Despite the large depolarization for F1A sample we measured ellipsometric angles and degree of polarization in many points on their surface and then we determined spectral dependences of optical indices $n(\lambda)$ and $k(\lambda)$. In Fig. 5, the dispersion of ellipsometric angles $\Psi$ and $\Delta$ has been shown. The red and green lines denote experimental data. The dotted lines are modelled curves, by the use of point-by-point Kramers-Kroning $(\mathrm{K}-\mathrm{K})$ relations [18]. The use of $\mathrm{K}-\mathrm{K}$ model allowed us to determine optical indices in the broad spectral range $(190$ to $1700 \mathrm{~nm})$. Figure 6 shows the $n(\lambda)$ and $k(\lambda)$ spectra obtained from the fitting of K-K model to experimentally measured ellipsometric parameters. The values determined by the ellipsometric tests (Fig. 4) overlap with the values obtained from the calculations made using the molecular luminance theory for 
$\lambda=532 \mathrm{~nm}$ (PALIK et al.). The BRDF method allows to describe surface topography in larger areas of spatial waves than SEM and AFM methods. For small roughness, the BRDF function is proportional to the spectral density of the roughness [19-22]. Experimentally determined BRDF functions based on spatial frequency for samples are shown in Figs. $7 \mathbf{a}$ and $7 \mathbf{b}$ for fly ash and metakaolin samples, respectively.

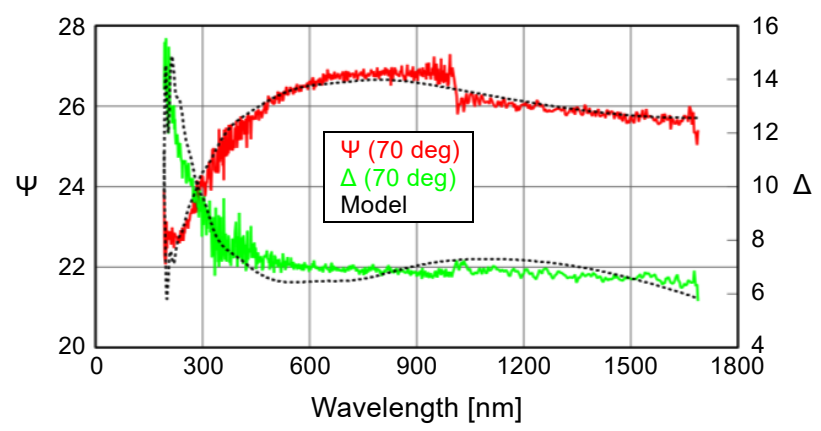

Fig. 5. Spectra of ellipsometric angles $\Psi$ and $\Delta$ for F1A sample.

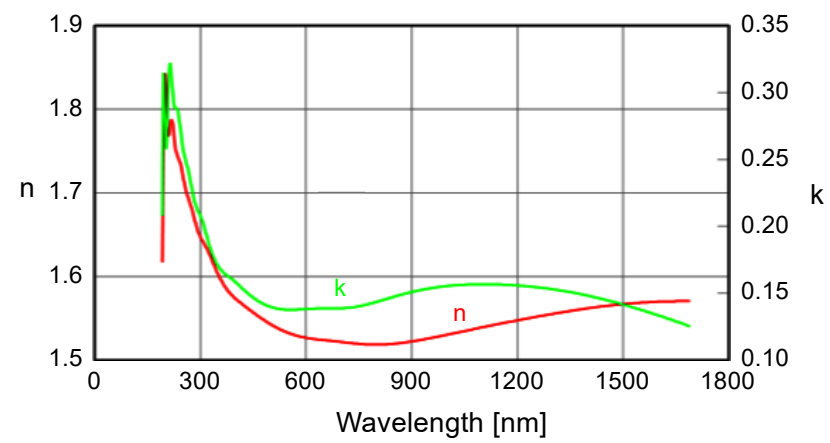

Fig. 6. Spectral dependence of refractive index $n(\lambda)$ and extinction coefficient $k(\lambda)$.
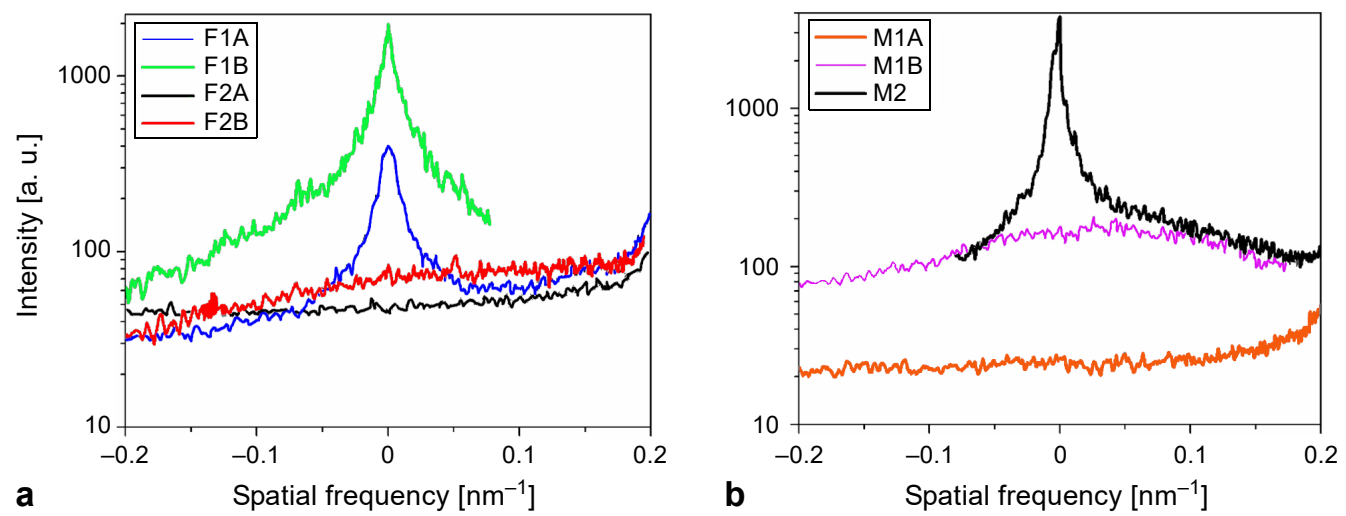

Fig. 7. BRDF as a function spatial frequency of geopolymer samples based on flying ashes (a), and metakaolin (b). 
In the BRDF spatial frequency spectra for metakaolin-based samples the peak occurs only for metakaolin 2 sample. Also it has been observed that samples based on metakaolins M1B and M2 created by the use of polypropylene molds, are more similar in shape than those for polycarbonate forms (M1A). Because surfaces from polypropylene molds were more flat, thus we observed the specular reflection. For metakaolin samples created by the use of polycarbonate forms, a full diffusive scattering on GP surface is observed (see Fig. 7). Strongly light scattering surfaces reflect the radiation in a completely incoherent way. Thus, there are no extremes in BRDF characteristics. For the so-called Lambertian surfaces ideal diffusers, the BRDF should be independent of spatial frequencies. However GP surfaces and most real diffusers are rather Seliger diffusers. Such surfaces also meet Seliger's law [10],

$$
I_{\mathrm{s}}=K \frac{\cos \theta_{\mathrm{i}} \cos \theta_{\mathrm{s}}}{\cos \theta_{\mathrm{i}}+\cos \theta_{\mathrm{s}}}
$$

where $K$ is the constant dependent on the absorption coefficient $k$ of the surface.

Deviation of Lambert's law for GP surfaces increases with the increase in the angle of incidence $\theta_{\mathrm{i}}$ and the scattering angle $\theta_{\mathrm{s}}$. For flying ashes samples, obtained in a polycarbonate form, we can see sharp maxima in BRDF spatial frequency dependences. Also for polypropylene molds, the surfaces are similar to Lambert diffusors. For better descriptions of light scattering, the choice of Seliger relation is most reasonable. For flying ashes samples, the results were completely different. Peaks, related to the directional reflection, were observed for samples in polycarbonate molds, for metakaolin -based samples this effect was not visible. Observation of SEM pictures showed that SEM is not suitable to describe such samples. Samples of the same source material and different molds do not differ much. They are fairly similar and - depending on the place where picture is taken, it is easy to mistake samples. In case of metakaolin samples it is actually impossible to recognize samples just by the SEM pictures. Fly-ash-based samples differ more, however those differences are related more to the fact that fly-ash -based geopolymers are not as homogenous as metakaolin-based ones, and depending on what part of a sample we examine the results may differ. However we can find places on the surfaces of the samples that are similar to places on the surfaces of the samples from different molds. That proves that analyzing photos, both from SEM and from optical microscopy (not shown in this paper), are not sufficient to study geopolymer samples surfaces. Ellipsometric measurements were difficult to perform due to the large GP surface roughness. Although depolarization of the VIS range was relatively low, it allowed us to make a measurement. The $\mathrm{K}-\mathrm{K}$ model is fitted to the surface for reflection from a single surface. Also for the other two GP surfaces, ellipsometric tests were performed. For the M1A surface, the values of the determined ellipsometric parameters measured at different points of the sample surface were reproducible. Although the ellipsometry test for the F1 and F2 surfaces in which the specular reflection occurs, we decided not include these results in this paper because of their low reproducibility. Lack of repeatability of results for distant points $(1 \mathrm{~mm})$ means that glossy surfaces are char- 
acterized by low autocorrelation coefficients. Knowledge of the spectral relationships of $n$ and $k$ allows modeling of light scattering from real surfaces for VIS and NIR.

\section{Summary and possibilities of application}

The surface features (reflective, matte) are important parameters, not only utilitarian, but also decorative (color and design) aspect. Various forms can be applied (depending on the requirements) to create a matte or reflective surfaces with a variable coefficient of gloss. Foil forms have allowed the weak, noticeable to naked eye specular reflectance. Also polypropylene molds are allowed to obtain matte surface.

\section{Conclusion}

Optical methods used in the paper were very effective to examine geopolymer samples and to measure their optical properties. Moreover we can see, that pictures from SEM or optical microscopy are not sufficient enough to complex examination of samples. They can only be used as supplementary to other optical methods such as TIS, BRDF, ellipsometry, etc. It was proven that a mold type has a significant impact on results achieved. Moreover results differ depending on source material and dye used but only in the VIS range. It is interesting that total reflectance that decreases for fly-ash-based geopolymers in most cases it increases for metakaolin-based geopolymers. Optical methods may have a decisive influence on the assessment of the utility of the geopolymers as a façade material or coating. Optical methods are non-destructive and in some cases they could be used in situ, on the construction site. That means that optical methods could be most important methods to evaluate the suitability of geopolymers in applications, where material appearance (look) is significant, like coatings or covering materials.

\section{References}

[1] Davidovits J., Geopolymer Chemistry and Applications, 2nd Ed., Institut Géopolymère, 2008.

[2] Provis J.L., Van Deventer J.S.J., Geopolymers. Structures, Processing, Properties and Industrial Applications, Woodhead Publishing Series in Civil and Structural Engineering, 2009, pp. 464, DOI: $10.1533 / 9781845696382$.

[3] Pacheco-Torgal F., Labrincha J.A., Leonelli C., Palomo A., Chindaprasirt P., Handbook of Alkali-Activated Cements, Mortars and Concretes, Vol. 53, 2013, DOI: 10.1016/C2013-0-16511-7.

[4] Duxson P., Fernández-Jiménez A., Provis J.L., Lukey G.C., Palomo A., Van Deventer J.S.J., Geopolymer technology: the current state of the art, Journal of Materials Science 42, 2007, pp. 2917 -2933, DOI: 10.1007/s10853-006-0637-z.

[5] Provis J.L., Van Deventer J.S.J., Alkali Activated Materials, Vol. 13, Springer, Dordrecht, 2014, DOI: $10.1007 / 978-94-007-7672-2$.

[6] Vickers L., Van Riessen A., Rickard W.D.A., Fire-Resistant Geopolymers. Role of Fibres and Fillers to Enhance Thermal Properties, Springer, Singapore, 2015, DOI: 10.1007/978-981-287-311-8.

[7] Korniejenko K., Frączek E., Pytlak E., Adamski M., Mechanical properties of geopolymer composites reinforced with natural fibers, Procedia Engineering 151, 2016, p. 388-393, DOI: 10.1016 / j.proeng.2016.07.395. 
[8] Davidovits P.J., 30 Years of successes and failures in geopolymer applications. Market trends and potential breakthroughs, Geopolymer 2002 Conference, October 28-29, 2002, Melbourne, Australia, pp. 1-16.

[9] Peiponen K.-E., Myllylä R., Priezzhev A.V., Optical Measurement Techniques. Innovations for Industry and the Life Sciences, 1st Ed., Springer, Berlin, Heidelberg, 2005, DOI: 10.1007/978-3-540 -71927-4.

[10] Workman J., Jr., Preface, [In] Applied Spectroscopy, Workman J., Jr., Springsteen A.W., Academic Press, 1998, DOI: 10.1016/B978-012764070-9/50001-9.

[11] Rönnow D., Bergkvist M., Roos A., Ribiing C.G., Determination of interface roughness by using a spectroscopic total-integrated-scatter instrument, Applied Optics 32(19), 1993, pp. 3448-3451, DOI: $10.1364 / \mathrm{AO} .32 .003448$.

[12] Detrio J.A., Miner S.M., Standardized total integrated scatter measurements of optical surfaces, Optical Engineering 24(3), 1985, p. 243419, DOI: 10.1117/12.7973499.

[13] TAY C.J., WANG S.H., QuAN C., NG C.K., Surface roughness measurement of semi-conductor wafers using a modified total integrated scattering model, Optik 113(7), 2002, pp. 317-321, DOI: 10.1078/ 0030-4026-00169.

[14] Nicodemus F.E., Richmond J.C., Hsia J.J., Ginsberg I.W., Limperis T., Geometrical Considerations and Nomenclature for Reflectance, U.S. Government Printing Office, 1977, pp. 1-52.

[15] Stover J.C., Optical Scattering: Measurement and Analysis, 2nd Ed., SPIE Optical Engineering Press, 1995, DOI: $10.1117 / 3.203079$.

[16] Scheer B.W., Stover J.C., Development of a smooth-surface microroughness standard, Proceedings of SPIE 3141, 1997, pp. 78-87, DOI: 10.1117/12.287807.

[17] Elson J.M., Bennett J.M., Stover J.C., Wavelength and angular dependence of light scattering from beryllium: comparison of theory and experiment, Applied Optics 32(19), 1993, pp. 3362-3376, DOI: 10.1364/AO.32.003362.

[18] Lucarini V., Peiponen K.-E., SaArinen J.J., Vartiainen E.M., Kramers-Kronig Relations in Optical Materials Research, Springer, Berlin, Heidelberg, 2005, p. 27, DOI: 10.1007/b138913.

[19] Jaglarz J., Szewczenko J., Marszalek K., Basiaga M., Marszalek M., Gawee R., Nonstandard optical methods as a tool for rough surface analysis, Materials Today: Proceedings 2(7), 2015, pp. 4046-4052, DOI: 10.1016/j.matpr.2015.08.034.

[20] Jaglarz J., Wolska N., Mitura K., Duraj R., Marszalek K.W., El Kouari Y., Surface properties of hard protective coatings studied by optical techniques, Optical Materials 56, 2016, pp. 134-139, DOI: $10.1016 /$ j.optmat.2016.03.038.

[21] Jaglarz J., Topography descriptions of thin film by optical Fourier transform, Thin Solid Films 516(22), 2008, pp. 8077-8081, DOI: 10.1016/j.tsf.2008.04.072.

[22] Jaglarz J., Wagner T., Cisowski J., Sanetra J., Ellipsometric studies of carbazole-containing polymer layers, Optical Materials 29(7), 2007, pp. 908-912, DOI: 10.1016/j.optmat.2006.02.003. 